\title{
Attractive instability of oppositely charged membranes induced by charge density fluctuations
}

\author{
Guy Hed and S. A. Safran \\ Department of Materials and Interfaces, Weizmann Institute of Science, Rehovot 76100, Israel
}

\begin{abstract}
We predict the conditions under which two oppositely charged membranes show a dynamic, attractive instability. Two layers with unequal charges of opposite sign can repel or be stable when in close proximity. However, dynamic charge density fluctuations can induce an attractive instability and thus facilitate fusion. We predict the dominant instability modes and timescales and show how these are controlled by the relative charge and membrane viscosities. These dynamic instabilities may be the precursors of membrane fusion in systems where artificial vesicles are engulfed by biological cells of opposite charge.
\end{abstract}

The interactions between lipid bilayers control many biological processes such as membrane fusion, in which the domains separated by each bilayer merge [1]. In order to fuse, the bilayer must overcome an energetic barrier which can be reduced by an increase of the local bilayer curvature [2]. Thus, small wave length undulations of bilayers at close proximity promote fusion. We show here that electrostatic interactions between oppositely charged bilayers give rise to such fluctuations and predict the dynamics of instabilities related to the coupled distance and charge fluctuations. These dynamics may control the fusion of vesicles with oppositely charged cell membranes and our results indicate how the time scale for such fusion can be optimized as a function of the relative charges and viscosities.

The interaction of similarly charged surfaces is repulsive at the mean-field level and in the limit of relatively high salt concentration can be described by the DebyeHückel (DH) approximation to the Poisson-Boltzmann equation [3]. This repulsion between the surfaces inhibits surface undulations and thus opposes fusion, but fluctuations in the density of the charged lipids can sometimes lead to an instability, as was demonstrated theoretically [4] and numerically [5]. The case of oppositely charged bilayers, however, is qualitatively different because (as we show below) a system governed only by electrostatics is always unstable; the unstable mode may be related to charge fluctuations, height undulations, or a mixture of both, depending on the spacing between the layers, the charge densities and the viscosities.

Recent experiments show that positively charged lipidDNA complexes fuse with negatively charged cell membranes [6]. The subsequent release and transfection of DNA in such systems makes these complexes possible candidates for gene therapy. Understanding and control of the fusion process in this system of oppositely charged membranes is important so that one can minimize the time in which the complex is engulfed by the cell. In addition, once the complex is inside the cell it is surrounded by a positively charged bilayer that originated in the cell membrane. Another fusion of these oppositely charged membranes is needed in order to release the DNA into the cytoplasm [7]. Our predictions for the stability of the system as a function of the membrane charges and viscosities, may allow the optimization of the charge and viscosity of the lipid-DNA complex in order to enhance the fusion and transfection process.

Parsegian and Gingell [8] solved the PoissonBoltzmann equation using the $\mathrm{DH}$ approximation for two oppositely charged surfaces. We imagine that the charge density is controlled by mixing charged and neutral lipids; this is the case in both cellular membranes and in the lipid-DNA complexes. When the charge densities on the surfaces are different, there are counterions that must remain in the region between the surfaces in order to balance the electrostatic interaction. At close proximity, the pressure of these counterions leads to a net repulsion between the surfaces. For flexible surfaces, this short range repulsion inhibits undulations when the surfaces are at a small distance. Such undulations are needed in order for fusion to occur. Thus, within this simple picture in which the surface charges are uniform, oppositely charged surfaces do not necessarily attract and will not necessarily fuse. In this paper, we consider the effects of lateral fluctuations of the charges in each membrane and show that at relatively short distances, these fluctuations give rise to a dynamic instability that promotes fusion. The instability involves a coupled mode of the local charge densities and the distance between the bilayers.

In the DH limit, applicable to physiological salt concentrations, the screening length that governs the interaction between the bilayers is $\kappa^{-1}$, where $\kappa^{2}=\frac{8 \pi n_{\mathrm{s}} Q_{\mathrm{s}}^{2}}{\epsilon k_{\mathrm{B}} T}$, $n_{\mathrm{s}}$ is the salt concentration, $Q_{\mathrm{s}}$ is the charge per lipid and $\epsilon$ is the dielectric constant of water. The charge density of each membrane $i=1,2$ is $\sigma_{i} e / a$, where $a$ is the area of a lipid and $\sigma_{i}$ is a dimensionless charge density. The electrostatic free energy of the system per unit area is [3, 8]

$$
\begin{aligned}
f_{\mathrm{el}} & =\frac{2 \pi e^{2}}{\epsilon \kappa a^{2}} \times \\
& \left(\frac{\left(\sigma_{1}{ }^{2}+{\sigma_{2}}^{2}\right) \exp (-h)+2 \sigma_{1} \sigma_{2}}{\sinh (h)}+{\sigma_{1}}^{2}+{\sigma_{2}}^{2}\right),
\end{aligned}
$$

where $h$ is the dimensionless distance between the membranes in units of $\kappa^{-1}$. The last two terms in Eq. 1 account for the electrostatic repulsion between lipids in the same bilayer. 


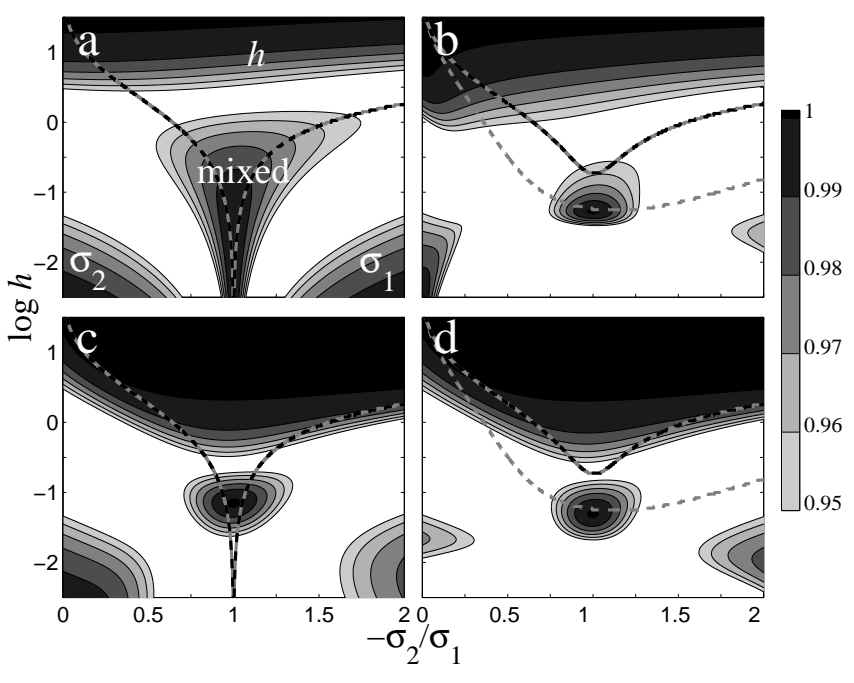

FIG. 1: The projections of the unstable eigenmodes of the system on the modes predicted for the limiting cases. (a) We use $f=f_{\text {el }}$ and $\sigma_{1}=0.8$ to calculate the energy stability matrix, $\boldsymbol{S}$, at each point on the $h \times \sigma_{2}$ surface. Let $\vec{u}$ be the eigenvector with the negative eigenvalue of $\boldsymbol{S}$. In each region the color code represents the projection $\vec{u} \cdot \vec{v}_{i}$, with $\vec{v}_{i}$ is $\vec{v}_{h}$, $\vec{v}_{\sigma_{1}}, \vec{v}_{\sigma_{2}}$ or $\vec{v}_{\mathrm{m}}$ (defined in the text), according to the label on that region. Below the black-grey dashed line $f_{h h}>0$ so a a system with fixed constant charge density profile is stable there. (b) The same, but for $f=f_{\text {el }}+f_{\text {ent }}$. Below the dashed gray line all the eigenvalues are positive, and there is no unstable mode. We choose $\vec{u}$ there as the eigenvector with the smallest eigenvalue. (c) The same as in (a), but for the unstable eigenvector of the dynamical matrix, $\boldsymbol{D}$, (see Eq. 5 ). (d) The same as in (b), but for the eigenvectors of $\boldsymbol{D}$.

We assume that the average distance between the bilayers is imposed by external constraints, such as lateral or transversal pressure, binding of trans-membranal proteins, or the system geometry, as in the case where the cell membrane encloses the lipid/DNA complex []]. Similarly, the overall charge on each membrane is conserved. We therefore focus only on local changes in the intermembrane spacing and charge density.

As a first step, we consider only the electrostatic free energy, that is, we let the total free energy, $f=f_{\mathrm{el}}$. In the calculations below, we assume $\sigma_{1}>-\sigma_{2}>0$. The thermodynamic stability (see below for the dynamics) of the system depends on the eigenvalues of the Hessian matrix $\boldsymbol{S}$ of the second variations of the free energy $f$,

$$
\boldsymbol{S}=\left(\begin{array}{ccc}
f_{h h} & f_{h \sigma_{1}} & f_{h \sigma_{2}} \\
f_{h \sigma_{1}} & f_{\sigma_{1} \sigma_{1}} & f_{\sigma_{1} \sigma_{2}} \\
f_{h \sigma_{2}} & f_{\sigma_{1} \sigma_{2}} & f_{\sigma_{2} \sigma_{2}}
\end{array}\right) .
$$

The determinant $|\boldsymbol{S}|=\left(\frac{4 \pi e^{2}}{\epsilon \kappa a^{2}}\right)^{3} \frac{\sigma_{1} \sigma_{2}}{\sinh (h)}$ is always negative for oppositely charged layers. In fact, $\boldsymbol{S}$ has one negative eigenvalue $\lambda_{i}$, which corresponds to the unstable eigenmode $\vec{v}_{i}$ of the system. We estimate this unstable mode analytically for three limiting cases:

(1) For $h \gg 1$ we have $\lambda_{h}=\frac{8 \pi e^{2}}{\epsilon \kappa a^{2}} \sigma_{1} \sigma_{2} \exp (-h)$ and $\vec{v}_{h}=(1,0,0)$. This represents a mode where there are surface undulations but with a constant charge density. This constant density is the result of the repulsion between similar charges on the same bilayer, which inhibits charge density fluctuations. In this case, the instability is due to the "naive" picture of attraction of oppositely (but uniformly) charged surfaces.

(2) When $h \ll 1$ and $h \ll \sigma_{1}+\sigma_{2}$ the non-diagonal terms of $\boldsymbol{S}$ vanish and $h, \sigma_{1}$ and $\sigma_{2}$ decouple. The unstable mode (under our assumption of $\left|\sigma_{2}\right|<\left|\sigma_{1}\right|$ ) is $\vec{v}_{\sigma_{2}}=(0,0,1)$ with $\lambda_{\sigma_{2}}=\frac{4 \pi e^{2}}{\epsilon \kappa a^{2}} \frac{\sigma_{2}}{\sigma_{1}+\sigma_{2}} h$ (in the case of $\left|\sigma_{2}\right|>\left|\sigma_{1}\right|$ the unstable mode is $\left.\vec{v}_{\sigma_{1}}=(0,1,0)\right)$. The associated density fluctuations tend to lead to regions where locally $\sigma_{1} \approx-\sigma_{2}[9]$. This is the situation in which the largest attractions occur since there is no need for counterions to balance the electrostatic interaction. Thus, the counterions may be depleted from these regions and the associated entropic repulsion between the layers vanishes. This situation, where the interaction takes the form of a nearly bare Coulomb attraction between surfaces, is energetically favorable. There are fluctuation only in $\sigma_{2}$ since the repulsive interactions between charges in the same bilayer inhibits charge fluctuations in the more highly charged bilayer $\left({\sigma_{2}}^{2}<{\sigma_{1}}^{2}\right)$ and the charge fluctuations are therefore limited to the bilayer with the lower charge density.

(3) When $\sigma_{1}+\sigma_{2} \ll h \ll 1$ the charge densities are almost equal and opposite, $\sigma_{1} \approx-\sigma_{2}$, and the interaction between the layers takes the form of an unscreened Coulomb attraction. Charge density variations serve to increase the instability. The eigenmode $\vec{v}_{\mathrm{m}}=\frac{1}{2}(\sqrt{2},-1,1)$ is an equal mixture of height undulations and in-phase charge density fluctuations. The energy associated with this instability is given by the eigenvalue $\lambda_{\mathrm{m}}=-\frac{\sqrt{2} \pi e^{2}}{\epsilon \kappa a^{2}}\left|\sigma_{1}-\sigma_{2}\right|$. In order to estimate the contribution of the charge fluctuations to this instability, we compare its energy scale, given by $\lambda_{\mathrm{m}}$, with the energy scale for the same instability of a system with uniform, local constant charge densities, given by $f_{h h}$. To zeroth order in $\sigma_{1}+\sigma_{2}$ we have $\frac{f_{h h}}{\lambda_{\mathrm{m}}}=\frac{\sigma_{1} \sinh (h / 2)}{\sqrt{2} \cosh ^{3}(h / 2)} \sim h \ll 1$. This means that charge fluctuations, when they are allowed, significantly increase the unstable eigenvalue and thus speed up the dynamical attractive instability.

In order to more accurately determine the regions of validity of these limiting cases, we calculated numerically the unstable eigenmodes of the stability matrix, $\boldsymbol{S}$, and their projections on each of the "ideal" modes found analytically in the limiting cases discussed above. As seen in Fig. पa, for $h \lesssim 1$ there is wide ranges of values of $\sigma_{2}$ where the mixed mode $\vec{v}_{\mathrm{m}}$ is dominant. The charge density modes $\vec{v}_{\sigma_{1}}$ and $\vec{v}_{\sigma_{2}}$ are dominant at $h \lesssim 0.25$ and $\left|1+\sigma_{2} / \sigma_{1}\right|>0.3$.

In addition to the electrostatic energy, charge fluctuations caused by the demixing of the charged and neutral lipids also modify the local entropy of each bilayer, given by $f_{i}=\frac{k_{\mathrm{B}} T}{a}\left[c_{i} \log c_{i}+\left(1-c_{i}\right) \log \left(1-c_{i}\right)\right]$ (for $\left.i=1,2\right)$, where $c_{i}=\sigma_{i} e / Q_{i}$ is the number density of the charged 
lipids in bilayer $i$, and $Q_{i}$ is the charge of a single lipid. The entropy will tend to remix the two species and may inhibit to some degree or even eliminate the charge instabilities that are promoted by the electrostatic interactions. On the other hand, attractive interactions, such as Van der Waals attraction between similar lipids, promote the demixing charge fluctuations and enhance the instability. Here we consider the "worst case" where there are no such attractions.

We also include the Helfrich repulsion between the bilayers, induced by the entropy of the bilayer undulations [10]. Its contribution to the free energy is $f_{\mathrm{H}}=$ $c_{\mathrm{H}}\left(\kappa k_{\mathrm{B}} T\right)^{2}\left(k_{1}^{-1}+k_{2}^{-1}\right) h^{-2}$, where $c_{\mathrm{H}} \simeq 0.116$ is a universal number, and $k_{i}$ is the bending modulus of bilayer $i$. The contribution of the entropy of the charged lipids and of the undulations to the free energy is $f_{\text {ent }}=f_{1}+f_{2}+f_{\mathrm{H}}$.

In Fig. 1b, we present results for the eigenmodes of the stability matrix, $\boldsymbol{S}$, for the total free energy $f=f_{\text {el }}+f_{\text {ent }}$. The smallest eigenvalue is positive in the regions where the $\vec{v}_{\sigma_{1}}$ and $\vec{v}_{\sigma_{2}}$ modes are dominant, and the system is stable there. Nevertheless, there is a finite range of $\sigma_{2}$ values, for which the mixed mode $\vec{v}_{\mathrm{m}}$ is dominant, where the system is unstable even for values of $h$ as small as 0.4. Thus, the entropy modifies, but does not eliminate entirely the predicted instabilities.

In order to test this prediction one can measure the stability of multilayer systems made of alternating layers of positive and negative charge. Systems that contain random mixtures of cationic and anionic lipids were already examined [11]. However, for the instabilities to be observed, the cationic and anionic lipids must phase separate (due to e.g., different chemistry of the polar heads or tails). The spacing between the layers can be determined by the fraction of water in the mixture. We predict that the system will be stable when the interlayer distance $h$ is small, but it will become unstable when $h$ is larger than a critical value $h_{\mathrm{c}}$ as shown in Fig. 1] which depends on the charge densities of the bilayers.

We now consider the dynamical response of the system in order to predict not just the regions of possible stability, but also the time scales associated with the distance and charge instabilities. These timescales and their dependence on the charge and the viscosities are important in controlling the fusion processes in the experimental systems described above. We therefore consider the dynamical response of the system to a small perturbation with a long wavelength $2 \pi q^{-1} \gg \bar{h}$, where $\bar{h}=\kappa^{-1} h$ is the local instantaneous distance between the membranes, and $q$ is the wave-number of the perturbation.

The hydrodynamics of the water layer between the bilayers in the approximation of long wavelength perturbations is given, to first order in $q \bar{h}$, by the lubrication approximation [12]:

$$
\eta \frac{\partial \bar{h}}{\partial t}=\frac{\partial}{\partial x}\left[\frac{\bar{h}^{3}}{3} \frac{\partial}{\partial x}\left(\frac{\partial f}{\partial \bar{h}}-\gamma \frac{\partial^{2} \bar{h}}{\partial x^{2}}\right)\right],
$$

where $\eta$ is the water viscosity and $\gamma$ is the membrane surface tension, which we can neglect if the wave number

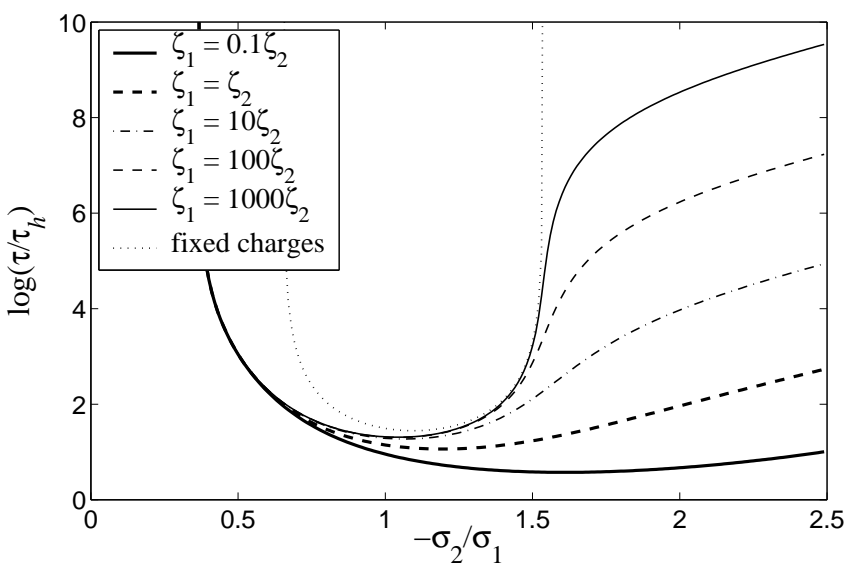

FIG. 2: The time scale $\tau$ for the growth of the instability for different values of $\zeta_{1} . \tau$ is given by inverse of the negative eigenvalue of the dynamical matrix, $\boldsymbol{D}$, calculated for $f=$ $f_{\text {el }}+f_{\text {ent }}, h=1$ and $\zeta_{2}=10^{-7}$ erg s $/ \mathrm{cm}^{2}$. We keep $\sigma_{1}=0.8$ and vary $\sigma_{2}$ along the $x$-axis. The dotted line is the time scale for a system with a uniform charge density. $\tau$ diverges when the system is stable.

$q$ is small enough (see discussion at the end of the letter).

The dynamics of the charged lipids in the membrane is governed by the Smoluchowski equation [13.

$$
\frac{\partial c_{i}}{\partial t}=\frac{\partial}{\partial x} \frac{1}{\zeta_{i}}\left(c_{i} \frac{\partial \mu_{i}}{\partial x}\right) ; \quad \mu_{i}=\frac{\partial f}{\partial c_{i}}
$$

where $\mu_{i}(i=1,2)$ is the chemical potential of a charged lipids in bilayer $i, c_{i}$ is the number density of the charged lipids and $\zeta_{i}$ is the two-dimensional viscosity in the bilayer.

We consider a perturbation of the type $h(x, t)=h_{0}+$ $\delta h(t) \sin (\vec{q} \cdot \vec{x}), \sigma_{i}(x, t)=\sigma_{i 0}+\delta \sigma_{i}(t) \sin (\vec{q} \cdot \vec{x})$. From Eqs. 3 and 4 we obtain the dynamics to first order in $\delta h$ and $\delta \sigma_{i}$ :

$$
\begin{gathered}
\frac{\partial}{\partial t}\left(\begin{array}{c}
\delta h \\
\delta \sigma_{1} \\
\delta \sigma_{2}
\end{array}\right)=-\boldsymbol{D}\left(\begin{array}{c}
\delta h \\
\delta \sigma_{1} \\
\delta \sigma_{2}
\end{array}\right) \\
\boldsymbol{D}=q^{2}\left(\begin{array}{ccc}
\frac{h^{3}}{3 \eta \kappa} & 0 & 0 \\
0 & \frac{Q_{1} a \sigma_{1}}{e \zeta_{1}} & 0 \\
0 & 0 & \frac{Q_{2} a \sigma_{2}}{e \zeta_{2}}
\end{array}\right) \boldsymbol{S},
\end{gathered}
$$

where the derivatives in $\boldsymbol{S}$ are evaluated at $h=h_{0}$ and $\sigma_{i}=\sigma_{i 0}$.

From Eq. 5 we obtain characteristic time scales for the dynamics $h, \sigma_{1}$ and $\sigma_{2}: \tau_{h}=3 \eta \kappa \frac{\epsilon \kappa a^{2}}{2 \pi e^{2}} q^{-2}, \tau_{\sigma_{i}}=$ $\frac{\zeta_{i}}{Q_{i}} \frac{\epsilon \kappa a}{2 \pi e} q^{-2}$. For the numerical calculation of the time scales we use the values: $\sigma_{1}=0.8, Q_{1}=-Q_{2}=2 e$, $\eta=0.01 \mathrm{erg} \mathrm{s} / \mathrm{cm}^{3}, \zeta_{1}=\zeta_{2}=10^{-7} \mathrm{erg} \mathrm{s} / \mathrm{cm}^{2}$ 14, $T=298 \mathrm{~K}, k_{1} \gg k_{2}=20 k_{\mathrm{B}} T, a=10^{-14} \mathrm{~cm}^{2}$ and $\kappa=10^{7} \mathrm{~cm}^{-1}$. With these values we have $\tau_{\sigma_{i}}=35 \tau_{h}$. If we consider $\epsilon=4 \pi \epsilon_{0} \epsilon_{\text {water }}=3.5 \cdot 10^{20} e^{2} \mathrm{erg}^{-1} \mathrm{~cm}^{-1}$ and $q=10^{5} \mathrm{~cm}^{-1}$ we have $\tau_{h}=1.7 \cdot 10^{-6} \mathrm{~s}$. 
The time scale for the growth of the instability is the inverse of the negative eigenvalue of the dynamical matrix, $\boldsymbol{D}$ (in the case that one exists). We have calculated the eigenmodes and time scales for the two cases we considered: (i) electrostatics alone, $f=f_{\mathrm{el}}$ (ii) electrostatics and entropy, $f=f_{\mathrm{el}}+f_{\mathrm{ent}}$. In Figs. 11 and 11 we present the regions where the unstable mode corresponds to one of the modes we found above for the limiting cases. The figures demonstrate that our results, obtained through analytical approximation, are valid for large regions in the parameter space.

When we take into account the entropy (Fig. 11) the mode $\vec{v}_{\sigma_{2}}$ almost vanishes and the regions in which the modes $\vec{v}_{\sigma_{2}}$ and $\vec{v}_{\sigma_{1}}$ were unstable in the absence of entropy, become stable. However, there is still a range of charge densities for which the system is destabilized by charge fluctuations. This range is represented in Fig. 11 by the area between the two stability lines. It is mostly dominated by the mixed mode $\vec{v}_{\mathrm{m}}$ that couples the charge density and distance fluctuations. This mixed mode results in a time scale for the attractive instability that is significantly faster than the time scale predicted from the mean-field attraction (i.e., in the absence of charge density fluctuations).

In Fig. 2 we present the dependence of the time scale $\tau$ for the instability on the intra-membrane viscosity $\zeta_{1}$. As expected, we see that if $\zeta_{1}>\zeta_{2}$ the time scales in the region where $\left|\sigma_{2}\right|>\left|\sigma_{1}\right|$, in which $\vec{v}_{\sigma_{1}}$ mode is dominant, are much slower than the time scales in the opposite region, where $\vec{v}_{\sigma_{2}}$ mode is dominant, and vice versa. An increase in the viscosity $\zeta_{1}$ shifts the maximal instability (and shortest time scale) to lower values of the charge ratio. This prediction indicates how one might optimize of the charge and viscosity values in the fusion and trans- fection experiments.

The electrostatic free energy $f_{\text {el }}$ promotes that growth of perturbations with any wave number $q$. These instabilities in are inhibited by the surface tension $\gamma_{i}$ and the bending modulus $k_{i}$ of each membrane labelled by $i=1,2$. For simplicity, we assume here that $k_{2}=k \ll k_{1}$ and $\gamma_{2}=\gamma \ll \gamma_{1}$, so that variations of the intermembrane distance, $h$, only arise from undulations of the more flexible bilayer, denoted as bilayer 2. In this case, the only contribution of these terms to the stability matrix, $\boldsymbol{S}$, (defined for the electrostatic-only case, $f=f_{\text {el }}$ ) will be in the entry $S_{11}=f_{\text {el } h h}+\gamma q^{2}+\frac{k}{2} q^{4}$, and $|\boldsymbol{S}|=\frac{16 \pi^{2} e^{4}}{\epsilon^{2} \kappa^{2} a^{4}}\left(\frac{4 \pi \sigma_{1} \sigma_{2} e^{2}}{\epsilon \kappa a^{2} \sinh (h)}+\gamma q^{2}+\frac{k}{2} q^{4}\right)$.

Let $q_{\max }$ be the wave number at which the system is marginally stable: $|\boldsymbol{S}|=0$. The system is unstable for all perturbations with longer wavelengths, $q<q_{\max }$. From Eq. 5 one sees that the time scale for the increase of a perturbation is proportional to $q^{-2}$. Thus the dominant instability of the system will be with a wave number $q \simeq$ $q_{\max }$.

At a small inter-membrane separation $h \ll 1$ we have $q_{\max } \sim h^{-\frac{1}{4}}$. As $h \rightarrow 0$ our theory predicts the growth of unstable modes with large wave vectors. The related undulations will grow until the bilayers are at a microscopic distance, where the electrostatic instability is balanced by short-range hydration repulsion. Thus, there will be regions where the bilayers are at close proximity and have high local curvature. In these regions the barrier for fusion is reduced considerably [2], and the probability for fusion is greatly increased.

The authors acknowledge useful discussions with C. R. Safinya, V. A. Parsegian and N. Kampf as well as the support of the Israel Science Foundation.
[1] E. Sackmann, in Handbook of Biological Physics, edited by R. Lipowsky and E. Sackman (Elsevier, Amsterdam, 1995), vol. 1B.

[2] Y. Kozlovsky and M. M. Kozlov, Biophys. J. 82, 882 (2002).

[3] D. Andelman, in Handbook of Biological Physics, edited by R. Lipowsky and E. Sackman (Elsevier, Amsterdam, 1995), vol. 1B.

[4] W. M. Gelbart and R. Bruinsma, Phys. Rev. E 55, 831 (1997).

[5] Y. W. Kim and W. Sung, Phys. Rev. Lett. 91, 118101 (2003).

[6] I. Koltover, T. Salditt, J. O. Radler, and C. R. Safinya, Science 281, 78 (1998).

[7] A. J. Lin, N. L. Slack, A. Ahmad, C. X. George, C. E. Samuel, and C. R. Safinya, Biophys. J. 84, 3307 (2003).

[8] V. A. Parsegian and D. Gingell, Biophys. J. 12, 1192 (1972).

[9] J. Nardi, R. Bruinsma, and E. Sackmann, Phys. Rev. E 58, 6340 (1998).

[10] R. Lipowsky, in Handbook of Biological Physics, edited by R. Lipowsky and E. Sackman (Elsevier, Amsterdam,
1995), vol. 1B.

[11] R. N. A. H. Lewis and R. N. McElhaney, Biophis. J. 79, 1455 (2000); S. R. Raghavan, G. Fritz, and E. W. Kaler, Langmuir 18, 3797 (2002); D. P. Pantazatos, S. P. Pantazatos, and R. C. MacDonald, J. Membrane Biol. 194, 129 (2003).

[12] A. Oron, S. H. Davis, and S. G. Bankoff, Rev. Mod. Phys. 69, 931 (1997).

[13] M. Doi and S. F. Edwards, The theory of polymer dynamics (Clarendon Press, Oxford, 1986), chap. 3, pp. 48-50.

[14] R. Merkel, E. Sackmann, and E. Evans, J. Phys. (Paris) 50, 1535 (1989). 